\title{
A clean signal reconstruction approach for coherently combining multiple radars
}

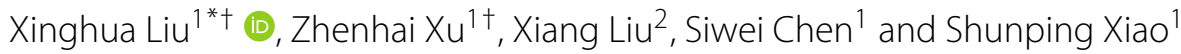

\begin{abstract}
Distributed radars have the potential to combine coherently for achieving a high signal-to-noise ratio (SNR) while maintaining a moderate antenna size. The key to coherently combining multiple radars is obtaining accurate coherent parameters (CPs), which are used to adjust the transmitting/receiving time and phase of each radar. One approach for CP estimation is to transmit orthogonal waveforms. However, ideally, orthogonal waveforms occupying the same frequency band may not be found in practice. Cross-correlation energy leakage exists between non-orthogonal waveforms, which seriously impairs the accurate acquisition of CPs. To solve this problem, we propose a clean signal reconstruction approach for $\mathrm{CP}$ estimation. This approach reconstructs clean echoes by gradually stripping out the cross-correlation energy leakage with a reconstruction-elimination-reconstruction framework. And CPs are obtained from these reconstructed clean echoes. Since the majority of cross-correction energy leakages are eliminated, enhanced CP estimation performance can be achieved. Verified simulations are designed for a dual radar scenario. Results show that the proposed approach significantly improves the performance of CP estimation while reducing the SNR requirement for coherently combining multiple radars.
\end{abstract}

Keywords: Multi-radar coherent combination, Coherent parameters, Clean signal reconstruction, All-pole model

\section{Introduction}

A single radar has a theoretical maximum signal-to-noise ratio (SNR) for a given size target at a given range, which directly affects radar's ability to detect, track, and identify the target [1]. There are mainly two solutions to raise SNR. One is to develop highly sensitive radar with a large antenna. However, such large antenna systems are costly and not easily transportable. Alternatively, by jointly processing the transmitted and received signals from several co-located small independent radars, it is possible to combine them into a coherently functioning system for a significantly enhanced SNR [1-4]. When $N$ radars are coherently combined, an $N^{3}$ SNR gain over a single radar can be acquired, i.e., full coherence is achieved on both transmitting and receiving [5].

Coherently combining multiple radars is challenging, especially wideband radars. Signal processing in terms of timing and phase adjustments should be carried out to

\footnotetext{
*Correspondence: xinghua217@163.com

${ }^{\dagger}$ Xinghua Liu and Zhenhai Xu contributed equally to this work.

'State Key laboratory of CEMEE, National University of Defence Technology,

Yanwachi 137, Changsha, People's Republic of China

Full list of author information is available at the end of the article
}

calibrate the incoherence induced by disparate propagation paths and synchronization errors. If relative positions of the radars are known to a fraction of a wavelength, accurate time delay or phase corrections can be implemented in accordance with this prior knowledge [2, 6]. However, measuring accurate relative positions is difficult and subject to increasing errors as the separation of radar antennas increases, if the radars are not relatively fixed [1]. Furthermore, only knowing the relative separation between the radars is not sufficient for multiple radars' coherent combination, since it does not account for the internal electrical difference caused by synchronization errors. Hence, an additional calibration process is needed.

Another approach relies upon periodically separating the monostatic and bistatic echoes at each radar [3]. The monostatic echo corresponds to its transmitted radar signal, while the bistatic echo corresponds to a transmitted radar signal from the other radar. By comparing the relative time and phase relationship between these returns, coherence parameters (CPs), i.e., fine time and phase correcting values, can be estimated. These parameters are then used to adjust the transmitting/receiving time and 
phase of each radar for obtaining the full coherence SNR gain. A key feature of this approach is that relative positions of radar are not critical and coherently combining multiple radars is achieved via target-based calibration. Inspired by such idea, relevant works are springing up, including CPs' Cramer-Rao bound (CRB) and the theoretical performance bounds analyze [7-9], scheme for dual radar coherent combination based on stepped-frequency signal [10-12], and dual radar coherent combination laboratory and field experiments $[13,14]$.

To separate the monostatic and bistatic returns, two different ways have been reported: keeping these echoes separated in the time domain, i.e., using time-division multiplexing (TDM) technique [1] or using orthogonal waveforms [2,3]. To note, the scattering response of the target is commonly time-varying and using TDM techniques will undermine the coherence among separated echoes. Hence, the latter approach is a better choice. When orthogonal waveforms are transmitted to estimate CPs, the monostatic and bistatic echoes can be separated by matched filtering. Conceptually, these orthogonal waveforms should have the same operating bandwidth and center frequency. Otherwise, $\mathrm{CP}$ estimates may be subject to additional errors due to the frequency-selective target scattering response. Aside from the common spectral requirement, the waveforms must be as nearly orthogonal as possible. However, it is hard to find ideally orthogonal waveforms occupying the same frequency band in practice. Cross-correlation energy leakage between nonideally ones cannot be ignored, which will affect the accurate acquisition of CPs.

In this paper, to the best of our knowledge, the influence of non-orthogonal waveforms on $\mathrm{CP}$ estimation is analysed in detail for the first time. Theoretical analyses demonstrate that the cross-correlation energy leakage stemmed from waveform non-orthogonality will introduce additional estimation errors and problems. Since the cross-correlation energy leakage is the dominant reason for impairing $\mathrm{CP}$ estimates, a $\mathrm{CP}$ estimation approach based on clean signal reconstruction is proposed. The basic idea of this approach is to reconstruct the clean signal that is not or rarely interfered by cross-correlation energy leakage. Once the clean single is reconstructed, improved CP estimates can be obtained via these reconstructed clean signals. And the effectiveness of our proposed approach is verified by a dual radar coherent combination example transmitting up- and down-chirp waveforms to estimate CPs.

The remainder of this paper is organized as follows. Section 2 introduces the principle of coherently combining multiple radars. Section 3 presents the CP estimation framework and discusses the influence of nonorthogonal waveforms on $\mathrm{CP}$ acquisition. The clean signal reconstruction approach for estimating $\mathrm{CPs}$ and validated simulations are detailed in Sections 4 and 5. Conclusions are given in Section 6.

\section{Principle of coherently combining multiple radars}

As radars are distributed, theoretically, each radartransmitted signal cannot superimpose coherently at the target due to disparate propagation paths from radars to the target and synchronization errors (The difference of target scattering characteristics among different radars can be approximately neglected, since radars are assumed to be nearly co-located compared to the target detection range). For the same reason, incoherence also exists among the echoes received by radars. And such incoherence among signals can be characterized as time and phase misalignments. Hence, to coherently combine multiple radars, i.e., achieve both coherent-on-transmit and coherent-on-receive, signal processing concerning time and phase adjustments should be carried out to calibrate the incoherence induced by the above reasons. Put it more concretely, if the signal transmitted by radar 1 (reference radar) arrives at the target after the signal transmitted by radar 2, proper time and phase adjustments for radar 2 are needed to slow down and retard its transmitting time and phase appropriately.

Let $s\left(t-\kappa_{k}\right) e^{j 2 \pi f_{c}\left(t-\kappa_{k}\right)+j \varphi_{k}}$ denote the transmitted signal of radar $k, s(t)$ is the common baseband signal of all radars, $\kappa_{k}$ is the time synchronization error of radar $k$ compared to the reference clock, and $\varphi_{k}$ is the initial phase of radar $k$. Then, these signals propagated to the target can be expressed as

$$
s\left(t-\kappa_{k}-\tau_{k}\right) e^{j 2 \pi f_{c}\left(t-\kappa_{k}-\tau_{k}\right)+j \varphi_{k}}, k=1, \cdots, N
$$

where, $f_{c}$ is the carrier frequency, $\tau_{k}=R_{k} / c, R_{k}$ is the range from radar $k$ to the target (for the multi-scatterer target model case, $R_{k}$ denotes the range from radar $k$ to the target scattering centre), and $c$ is the speed of light.

Apparently, from (1), the arrival time and phase of these signals are misaligned. To enhance the radio frequency (RF) electric field impinging upon the target, i.e., achieve coherent-on-transmit, fine time and phase adjustments are necessary. Without loss of generality, if radar 1 is the reference radar, it is essential to adjust the transmitting time and phase of other radars. And the adjusted signals can be expressed as $s\left(t-\kappa_{k}+\Delta \tau_{k}^{t}\right) e^{j 2 \pi f_{c}\left(t-\kappa_{k}\right)+j \varphi_{k}} e^{-j \Delta \phi_{k}^{t},}$ $k=2, \cdots, N$, where

$$
\begin{aligned}
\Delta \tau_{k}^{t} & =\left(\tau_{k}-\tau_{1}\right)+\left(\kappa_{k}-\kappa_{1}\right) \\
\Delta \phi_{k}^{t} & =-2 \pi f_{c}\left[\left(\tau_{k}-\tau_{1}\right)+\left(\kappa_{k}-\kappa_{1}\right)\right]+\left(\varphi_{k}-\varphi_{1}\right)
\end{aligned}
$$

are the time and phase correcting values (transmit CPs) of radar $k$. To note, as modern radars are assumed to be used, 
in which digital waveform generators allow independent control of time and phase.

Let $u(t) e^{j 2 \pi f_{c} t}$ denote the reflected signal at the target, then this reflected signal received by radar $l$ after downconversion can be expressed as (assuming each radar can realize coherent reception)

$$
u\left(t-\tau_{l}+\kappa_{l}\right) e^{-j 2 \pi f_{c} \tau_{l}+j 2 \pi f_{c} \kappa_{l}-j \varphi_{l}}, l=1, \cdots, N
$$

For the same reason, to achieve coherent-onreceive, appropriate time and phase shifts are also needed for the echo received by other radars except radar 1. And the adjusting echo can be

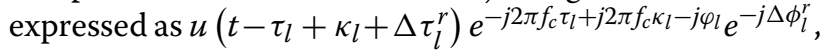
$l=2, \cdots, N$, where

$$
\begin{aligned}
& \Delta \tau_{l}^{r}=\left(\tau_{l}-\tau_{1}\right)-\left(\kappa_{l}-\kappa_{1}\right) \\
& \Delta \phi_{l}^{r}=-2 \pi f_{c}\left[\left(\tau_{l}-\tau_{1}\right)-\left(\kappa_{l}-\kappa_{1}\right)\right]-\left(\varphi_{l}-\varphi_{1}\right)
\end{aligned}
$$

denote the time and phase correcting values (receive CPs) of radar $l$. Note that the superscript $t$ is the abbreviation for "transmit," while the superscript $r$ is the abbreviation for "receive." Apparently, $\Delta \tau_{1}^{t}=\Delta \tau_{1}^{r}=$ $\Delta \phi_{1}^{t}=\Delta \phi_{1}^{r}$, the remaining CPs to be estimated are $\left\{\Delta \tau_{k}^{t}, \Delta \tau_{l}^{r}, \Delta \phi_{k}^{t}, \Delta \phi_{l}^{r}\right\}, k, l=2, \cdots, N$. For convenience, $\left\{\Delta \tau_{k}^{t}, \Delta \tau_{l}^{r}\right\}$ and $\left\{\Delta \phi_{k}^{t}, \Delta \phi_{l}^{r}\right\}$ are collectively called time and phase CPs.

\section{Problem formulation}

\subsection{Signal model and CP estimation framework}

Obviously, as the existence of synchronization errors, transmit and receive CPs of same radar are not identical. To estimate CPs, signals received by each radar have to be separated into monostatic and bistatic echoes firstly. It is the reason why orthogonal waveforms are transmitted. Assuming there is a target with Q-fixed non-scintillating scatterers in the space, in the state of each radar transmitting orthogonal waveforms, the signal received by radar $l$ after down-conversion can be expressed as (approximation is done based on the above assumption that radars are nearly co-located)

$$
\begin{aligned}
r_{l}(t) & \approx \sum_{q=1}^{Q} \sum_{k=1}^{N} \bar{\xi} s_{k}\left(t-\tau_{l k}-\Delta \tau^{q}\right) e^{-j 2 \pi f_{c}\left(\tau_{l k}+\Delta \tau^{q}\right)+j \varphi_{k}-j \varphi_{l}}+w_{l}(t) \\
& =\xi \sum_{k=1}^{N} s_{k}^{\prime}\left(t-\tau_{11}-\Delta \tau_{k}^{t}-\Delta \tau_{l}^{r}\right) e^{j \Delta \phi_{k}^{t}+j \Delta \phi_{l}^{r}}+w_{l}(t), l=1, \cdots, N
\end{aligned}
$$

where $\bar{\xi}^{q}$ denotes the scattering coefficient of scatterer $q$; $\tau_{l k}=\tau_{k}+\tau_{l}+\kappa_{k}-\kappa_{l}$ is the propagation time from radar $k$, to target scattering center, to radar $l ; \Delta \tau^{q}$ is the corresponding two-way propagation delay from scatterer $q$ to target scattering center in the radar line of sight (LOS); $s_{k}(t)$ denotes the orthogonal waveform transmitted by radar $k ; w_{l}(t)$ denotes the noise introduced during radar $l$ reception, which is assumed to be a zero-mean, complex white Gaussian random process; $s^{\prime}(t)=\sum_{q=1}^{Q} \bar{\xi}^{q} s_{k}$ $\left(t-\Delta \tau^{q}\right) e^{-j 2 \pi f_{c} \Delta \tau^{q}}$; and $\xi=e^{-j 2 \pi f_{c} \tau_{11}}$.

By matched filtering (MF), the $N$ received signals $\left\{r_{l}(t), l=1, \cdots, N\right\}$ can be separated into $N^{2}$ echoes. And each separated echo characterizes a distinct propagation path from radar $k$ to radar $l$, which can be formulated as

$$
\begin{aligned}
y_{l k}(t)= & r_{l}(t) \otimes s_{k}^{*}(-t) \\
= & \xi A_{k}^{\prime}\left(t-\tau_{11}-\Delta \tau_{k}^{t}-\Delta \tau_{l}^{r}\right) e^{j \Delta \phi_{k}^{t}+j \Delta \phi_{l}^{r}} \\
& +\xi \sum_{\substack{k^{\prime}=1 \\
k^{\prime} \neq k}}^{N} C_{k^{\prime} k}^{\prime}\left(t-\tau_{11}-\Delta \tau_{k^{\prime}}^{t}-\Delta \tau_{l}^{r}\right) e^{j \Delta \phi_{k^{\prime}}^{t}+j \Delta \phi_{l}^{r}} \\
& +w_{l k}^{\mathrm{MF}}(t), l, k=1, \cdots, N
\end{aligned}
$$

where $A_{k}^{\prime}(t)=\sum_{q=1}^{Q} \bar{\xi}^{q} A_{k}\left(t-\Delta \tau^{q}\right) e^{-j 2 \pi f_{c} \Delta \tau^{q}} ; C_{k^{\prime} k}^{\prime}(t)=$ $\sum_{q=1}^{Q} \bar{\xi}^{q} C_{k^{\prime} k}\left(t-\Delta \tau^{q}\right) e^{-j 2 \pi f_{c} \Delta \tau^{q}} ; A_{k}(t)$ is the autocorrelation function of $s_{k}(t)$; $C_{k k^{\prime}}(t)$ is the cross-correlation function between $s_{k^{\prime}}(t)$ and $s_{k}(t)$, i.e., the cross-correlation energy leakage term; and $w_{l k}^{\mathrm{MF}}(t)=w_{l}(t) \otimes s_{k}^{*}(-t), \otimes$ is the convolution operation, and $(\cdot)^{*}$ is the complex conjugate operation.

After separation, echo pair needs to be carefully selected to estimate CPs. For the dual radar case, the separated echo set is $\left\{y_{11}(t), y_{12}(t), y_{21}(t), y_{22}(t)\right\}$. Comparing $y_{11}(t)$ with $y_{12}(t)$, their receiving paths are identical, while transmitting paths are not. Hence, by comparing the time and phase differences between this echo pair, transmit CPs $\left\{\Delta \tau_{2}^{t}, \Delta \phi_{2}^{t}\right\}$ can be estimated. Meanwhile, comparing the echo pair $\left\{y_{21}(t), y_{22}(t)\right\}$, same transmit CPs can be also estimated. The ultimate transmit $\mathrm{CP}$ estimates will be the fusion of these two estimates. And the corresponding $\mathrm{CP}$ estimation framework is shown in Fig. 1, which can be easily extended to receive CP estimation and $N$-radar case.

On this basis, a CP estimator called cross-correlation (CC) processing is proposed $[3,10]$. In this estimator, the selected echo pair is cross-correlated. The peak of the cross-correlation output defines the corresponding time CPs, and the phase at the peak of the cross-correlation output defined the corresponding phase CPs. This processing can be briefly formulated as follows

$$
\begin{aligned}
& \Delta \hat{\tau}_{k}^{t}=\frac{1}{N} \sum_{l=1}^{N} \Delta \hat{\tau}_{k, l}^{t}, \Delta \hat{\phi}_{k}^{t}=\frac{1}{N} \sum_{l=1}^{N} \arg \left\{x_{k, l}^{t}\left(\Delta \hat{\tau}_{k, l}^{t}\right)\right\} \\
& \Delta \hat{\tau}_{l}^{r}=\frac{1}{N} \sum_{k=1}^{N} \Delta \hat{\tau}_{l, k}^{r}, \Delta \hat{\phi}_{l}^{r}=\frac{1}{N} \sum_{k=1}^{N} \arg \left\{x_{l, k}^{r}\left(\Delta \hat{\tau}_{l, k}^{r}\right)\right\}
\end{aligned}
$$

where $\Delta \hat{\tau}_{k, l}^{t}$ and $\Delta \hat{\tau}_{l, k}^{r}$ denote the peaks of echo pair crosscorrelation outputs $x_{k, l}^{t}(\tau)=\int_{T_{\mathrm{G}}} y_{l k}(t) y_{l 1}^{*}(t-\tau) d t$ and $x_{l, k}^{r}(\tau)=\int_{T_{\mathrm{G}}} y_{l k}(t) y_{1 k}^{*}(t-\tau) d t, T_{\mathrm{G}}$ is the time span of the radar receive range gate (see Fig. 1), and $\arg \{\cdot\}$ denotes the phase of a complex argument. 


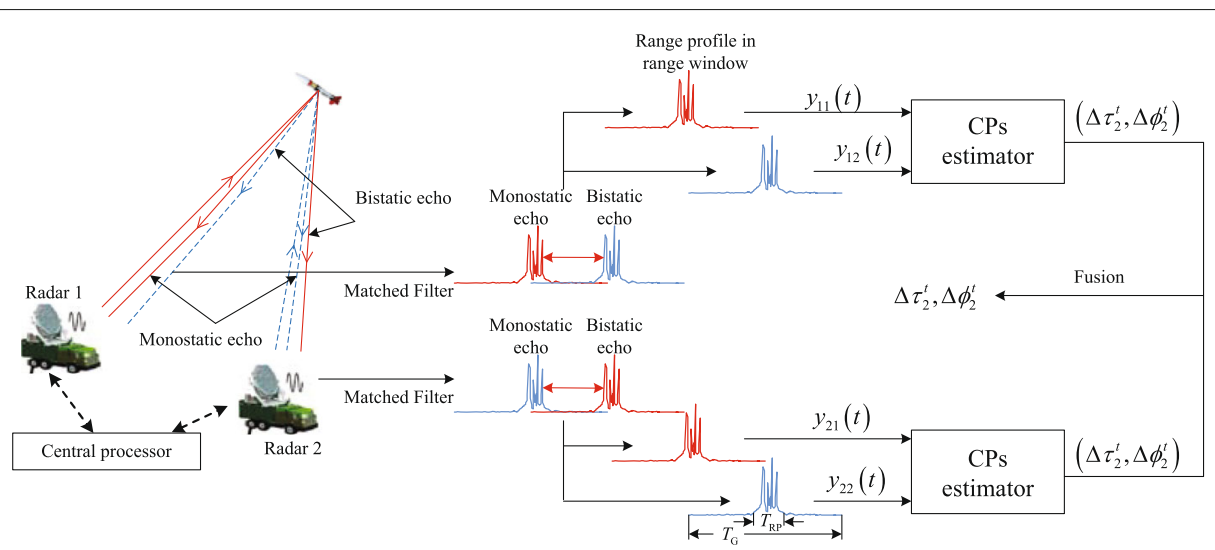

Fig. 1 Framework of transmit CP estimation for dual radar case

\subsection{Influence of transmitting non-orthogonal waveforms on $\mathrm{CP}$ estimation}

To note, CC is proposed on the assumption of transmitting ideally orthogonal waveforms. In practice, ideally orthogonal waveforms occupying same frequency band are not existent. In this case, $\mathrm{CP}$ estimation performance of CC may be impaired. And detailed analyses are illustrated in the following.

\subsubsection{Receive CP estimation}

Assuming SNR tends to infinity, from (6), $x_{l, k}^{r}(\tau)$ can be derived as $\left(|\xi|^{2}\right.$ is omitted)

$$
x_{l, k}^{r}(\tau)=e^{j \Delta \phi_{l}^{r}} \int_{T_{\mathrm{G}}} f_{k}\left(t-\Delta \tau_{l}^{r}\right)\left[f_{k}(t-\tau)\right]^{*} d t
$$

where $f_{k}(t)=A_{k}^{\prime}\left(t-\tau_{11}-\Delta \tau_{k}^{t}\right) e^{j \Delta \phi_{k}^{t}}+\sum_{\substack{k^{\prime}=1 \\ k^{\prime} \neq k}}^{N} C_{k^{\prime} k}^{\prime}$ $\left(t-\tau_{11}-\Delta \tau_{k^{\prime}}^{t}\right) e^{j \Delta \phi_{k^{\prime}}^{t}}$

Obviously, the peak of $x_{l, k}^{r}(\tau)$ is $\Delta \tau_{l}^{r}$, i.e., $\Delta \hat{\tau}_{l, k}^{r}=\Delta \tau_{l}^{r}$. By substituting $\Delta \hat{\tau}_{l, k}^{r}$ into (8), we have $x_{l k}^{r}\left(\Delta \hat{\tau}_{k, l}^{r}\right)=$ $M_{k}^{r} e^{j \Delta \phi_{l}^{r}}$, where $M_{k}^{r} \in \mathbb{R}$ denotes the effective estimation energy. Then, according to (7), we have

$$
\Delta \hat{\tau}_{l}^{r}=\Delta \tau_{l}^{r}, \Delta \hat{\phi}_{l}^{r}=\Delta \phi_{l}^{r}
$$

\subsubsection{Transmit CP estimation}

Under the same assumption, $x_{k, l}^{t}(\tau)$ can be derived as $\left(|\xi|^{2}\right.$ is omitted)

$$
\begin{aligned}
x_{k, l}^{t}(\tau)= & \int_{T_{\mathrm{G}}} e^{j \Delta \phi_{k}^{t}} A_{k}^{\prime}\left(t-\tau_{11}-\Delta \tau_{k}^{t}\right)\left[A_{1}^{\prime}\left(t-\tau_{11}-\tau\right)\right]^{*} \\
& +g_{k}(t, \tau) d t
\end{aligned}
$$

where $g_{k}(t, \tau)$ is the term related to cross-correlation energy leakage (for more details, see in Appendix A).
Divide (10) into two parts: the term with a peak at $\Delta \tau_{k}^{t}$ and the disturbance

$$
\begin{aligned}
x_{k, l}^{t}(\tau) \approx & \int_{T_{\mathrm{RP}}} e^{j \Delta \phi_{k}^{t}} A_{k}^{\prime}\left(t-\tau_{11}-\Delta \tau_{k}^{t}\right)\left[A_{k}^{\prime}\left(t-\tau_{11}-\tau\right)\right]^{*} d t \\
& +\underbrace{\int_{T_{\mathrm{G}}-T_{\mathrm{RP}}} e^{j \Delta \phi_{k}^{t}} A_{k}^{\prime}\left(t-\tau_{11}-\Delta \tau_{k}^{t}\right)\left[A_{1}^{\prime}\left(t-\tau_{11}-\tau\right)\right]^{*} d t+\int_{T_{\mathrm{G}}} g_{k}(t, \tau) d t}_{\text {disturbance }}
\end{aligned}
$$

where $T_{\mathrm{RP}}$ denotes the time span of the target range profile (RP) without "blank" margin (see Fig. 1). And in this time span, $A_{k}^{\prime}(t) \approx A_{1}^{\prime}(t)$.

Generally, the amplitude of $A_{k}(t)$ in the mainlobe region is far greater than that of $A_{k}(t)$ in the sidelobe region and $C_{k^{\prime} k}(t)$. Even if the disturbance exists, the peak of $x_{k, l}^{t}(\tau)$ will lie in the neighborhood of point $\Delta \tau_{k}^{t}$, that is

$$
\Delta \hat{\tau}_{k, l}^{t}=\Delta \tau_{k}^{t}+\varepsilon_{k},\left|\varepsilon_{k}\right| \in\left[0, \varepsilon_{k}^{\max }\right]
$$

To note, $\varepsilon_{k}^{\max } \geqslant 0$, whose value is determined by the selection of orthogonal waveforms $\left(A_{k}(t)\right.$ and $\left.C_{k^{\prime} k}(t)\right)$ and the geometric arrangement of radars $\left(\Delta \tau_{k}^{t}, k=2, \cdots, N\right)$.

By substituting (12) into (11), we have

$$
\begin{gathered}
x_{k, l}^{t}\left(\Delta \hat{\tau}_{k, l}^{t}\right)=\int_{T_{\mathrm{RP}}} e^{j \Delta \phi_{k}^{t}} A_{k}^{\prime}\left(t-\tau_{11}-\Delta \tau_{k}^{t}\right)\left[A_{k}^{\prime}\left(t-\tau_{11}-\Delta \tau_{k}^{t}-\varepsilon_{k}\right)\right]^{*} d t \\
+J_{k}^{t}\left(\varepsilon_{k}\right)=e^{j \Delta \phi_{k}^{t}} E_{k}^{t}+F_{k}^{t}\left(\varepsilon_{k}\right)+J_{k}^{t}\left(\varepsilon_{k}\right)
\end{gathered}
$$

where $E_{k}^{t}<\int_{T_{\mathrm{RP}}} A_{k}^{\prime}\left(t-\tau_{11}-\Delta \tau_{k}^{t}\right)\left[A_{k}^{\prime}\left(t-\tau_{11}-\Delta \tau_{k}^{t}\right)\right]^{*} d t$ $\in \mathbb{R}$ denotes the effective estimation energy, $F_{k}^{t}\left(\varepsilon_{k}\right) \in \mathbb{C}$ denotes the disturbance caused by peak position deviation, and $J_{k}^{t}\left(\varepsilon_{k}\right)=\int_{T_{\mathrm{G}}-T_{\mathrm{RP}}} A_{k}^{\prime}\left(t-\tau_{11}-\Delta \tau_{k}^{t}\right)$ $\left[A_{1}^{\prime}\left(t-\tau_{11}-\Delta \tau_{k}^{t}-\varepsilon_{k}\right)\right]^{*} d t+\int_{T_{\mathrm{G}}} g_{k}\left(t, \Delta \tau_{k}^{t}+\varepsilon_{k}\right) d t \in \mathbb{C}$ denotes the disturbance introduced by cross-correlation energy leakage and the mismatch between $A_{1}^{\prime}(t)$ and $A_{k}^{\prime}(t)$.

Combining (7), (12), and (13), we have 
$\Delta \hat{\tau}_{k}^{t}=\Delta \tau_{k}^{t}+\varepsilon_{k}, \Delta \hat{\phi}_{k}^{t}=\Delta \phi_{k}^{t}+\arg \left[F_{k}^{t}\left(\varepsilon_{k}\right)\right]+\arg \left[J_{k}^{t}\left(\varepsilon_{k}\right)\right]$.

Throughout the above analyses, transmitting nonorthogonal waveforms seriously affect the estimation performance of CC. And such influences can be outlined as follows:

(1) When SNR $\rightarrow \infty$, using CC, the estimated receive CPs are unbiased (see (9)), while the estimated transmit CPs are not (see (14)). And their estimated biases are determined by the selection of orthogonal waveforms and the geometric arrangement of radars;

(2) Since $T_{\mathrm{G}}>T_{\mathrm{RP}}$, it is easy to verify that $M_{k}^{r}>E_{k}^{t}$, which implies that estimating transmit CPs is more noise-sensitive than estimating receive CPs.

\section{Methods}

As analyzed in Section 3, cross-correlation energy leakages between non-orthogonal waveforms seriously impair the accurate acquisition of CPs. If such energy leakages can be eliminated before $\mathrm{CP}$ estimation, i.e., reconstruct clean signals and use them to estimate CPs, the impacts introduced by non-orthogonal waveforms will disappear. Inspired by this, a new approach for estimating CPs is proposed in this section. In the following, we first introduce the clean signal reconstruction framework. Then, an additional process called pre-match processing is elaborated as an enhancement of our proposed approach. Finally, we show the overall CP estimation process.

\subsection{Clean signal reconstruction}

From (6), since cross-correlation energy leakages in the separated echo originate from the presence of echoes of other waveforms in the mixed reception signal, by eliminating these echoes from the initial reception signal, a clean separated echo can be reconstructed. Specifically, take the clean reconstruction of $y_{l N}(t)$ as an example, cross-correlation energy leakages introduced from the echoes of $\left\{s_{1}(t), \cdots, s_{N-1}(t)\right\}$ in $y_{l N}(t)$ can be suppressed by reconstructing and eliminating these echoes from the initially mixed reception signal $r_{l}(t)$. Under this consideration, a clean signal reconstruction approach is designed with a reconstruction-elimination-reconstruction (R-E-R) framework, which can be illustrated as follows:

1. Reconstruction (the former one). MF is implemented on $r_{l}(t)$ in the frequency domain to separate the echo of $s_{1}(t)$. The separated echo, i.e., the spectrum of $y_{l 1}(t)$, will be

$$
\begin{aligned}
& Y_{l 1}(f)=R_{l}(f) \cdot S_{1}^{*}(f) \\
& =\xi \sum_{q=1}^{Q} \xi^{q} e^{-j 2 \pi f \Delta \tau^{q}} e^{-j 2 \pi f_{c} \Delta \tau} \cdot\left|S_{1}(f)\right|^{2} e^{-j 2 \pi f\left(\tau_{11}+\Delta \tau_{1}^{t}+\Delta \tau_{l}^{r}\right)} e^{j \Delta \phi_{1}^{t}+j \Delta \phi_{l}^{r}} \\
& +S_{1}^{*} f(f)\left[\sum_{k^{\prime}=2}^{N} V_{l k^{\prime}}(f)+W_{l}(f)\right]
\end{aligned}
$$

where $f \in[-B / 2, B / 2], B$ is the bandwidth of the orthogonal waveforms, $V_{l k^{\prime}}(f)=\xi \sum_{q=1}^{Q} \bar{\xi}^{q} e^{-j 2 \pi f \Delta \tau^{q}}$ $e^{-j 2 \pi f_{c} \Delta \tau^{q}} \cdot S_{k^{\prime}}(f) e^{-j 2 \pi f\left(\tau_{11}+\Delta \tau_{k^{\prime}}^{t}+\Delta \tau_{l}^{r}\right)} e^{j \Delta \phi_{k^{\prime}}^{t}+j \Delta \phi_{l}^{r}}$ is the cross-correlation energy leakage term, $R_{l}(f)$, $S_{k}(f)$, and $W_{l}(f)$ are the spectrum of $r_{l}(t), s_{k}(t)$, and $w_{l}(t)$, respectively.

At high frequencies, according to the geometrical diffraction theory (GDT), the radar backscatter from a target can be accurately represented by an all-pole model [15-17]. Let $X_{l 1}(f)=Y_{l 1}(f) /\left|S_{1}(f)\right|^{2}$ (remove the effect of waveform spectral envelop on solving all-pole model). After discretization, $X_{l 1}(f)$ can be formulated as

$$
\begin{aligned}
X_{l 1}\left(f_{m}\right)= & \underbrace{\sum_{q=1}^{Q} d_{l 1}^{q}\left(p_{l 1}^{q}\right)^{m}}_{\text {all-pole model }} \\
& +\frac{S_{1}^{*}(m)}{\left|S_{1}(m)\right|^{2}}\left[\sum_{k^{\prime}=2}^{N} V_{l k^{\prime}}(m)+W_{l}(m)\right]
\end{aligned}
$$

where $d_{l 1}^{q}=\xi \bar{\xi}_{q} e^{j \pi B\left(\tau_{11}+\Delta \tau_{1}^{t}+\Delta \tau_{l}^{r}+\Delta \tau^{q}\right)} e^{-j 2 \pi f_{c} \Delta \tau^{q}}$ $e^{j \Delta \phi_{1}^{t}+j \Delta \phi_{l}^{r}}$ and $p_{l 1}^{q}=e^{-j 2 \pi \Delta f\left(\tau_{11}+\Delta \tau_{1}^{t}+\Delta \tau_{l}^{r}+\Delta \tau^{q}\right)}$ are the poles and their amplitudes, $f_{m}=-B / 2+m \Delta f$, $m=1, \cdots, M-1, \Delta f=B / M$. By solving the parametric model in (16), $Y_{l 1}(f)$ can be reconstructed as

$$
\hat{Y}_{l 1}^{c}\left(f_{m}\right)=\sum_{q=1}^{\hat{Q}_{l 1}} \hat{d}_{l 1}^{q}\left(\hat{p}_{l 1}^{q}\right)^{m}\left|S_{1}\left(f_{m}\right)\right|^{2}
$$

where $\hat{d}_{l 1}^{q}, \hat{p}_{l 1}^{q}$, and $\hat{Q}_{l 1}^{q}$ are the solved parameters (more details in Appendix B);

2. Elimination. Although the solved parameters in (17) are contaminated by $V_{k^{\prime}}(m), k^{\prime}=2, \cdots, N, \hat{Y}_{l 1}^{c}\left(f_{m}\right)$ still reserves the majority energy of $Y_{l 1}\left(f_{m}\right)$. Generally speaking, $\hat{Y}_{l 1}^{c}\left(f_{m}\right)$ can be considered as an approximation of $Y_{l 1}\left(f_{m}\right)$ in the least-squares sense. To note, the superscript $c$ in $\hat{Y}_{l 1}^{c}\left(f_{m}\right)$ represents the word "contaminated." And the echo of $s_{1}(t)$ in $r_{l}(t)$ can be reconstructed as $\hat{Y}_{l 1}^{c}\left(f_{m}\right) S_{1}\left(f_{m}\right) /\left|S_{1}\left(f_{m}\right)\right|^{2}$. Then, by eliminating it from the initially received mixed signal, its introduced cross-correlation energy leakage will be suppressed. This process is formulated as

$$
\left[Y_{l 1}\left(f_{m}\right)-\hat{Y}_{l 1}^{c}\left(f_{m}\right)\right] \frac{S_{1}\left(f_{m}\right)}{\left|S_{1}\left(f_{m}\right)\right|^{2}}
$$

where the product term $S_{1}\left(f_{m}\right) /\left|S_{1}\left(f_{m}\right)\right|^{2}$ denotes the inverse MF processing contrary to multiplying $S_{1}^{*}\left(f_{m}\right)$. 
By multiplying (18) with $S_{2}^{*}\left(f_{m}\right)$, the echo of $s_{2}(t)$ can be separated

$$
\tilde{Y}_{l 2}\left(f_{m}\right)=\left[Y_{l 1}\left(f_{m}\right)-\hat{Y}_{l 1}^{c}\left(f_{m}\right)\right] \frac{S_{1}\left(f_{m}\right)}{\left|S_{1}\left(f_{m}\right)\right|^{2}} S_{2}^{*}\left(f_{m}\right) .
$$

To distinct with the separated echo $Y_{l 2}\left(f_{m}\right)$ via direct $M F$, this one is tilded. With the same process, the echo of $s_{2}(t)$ in $r_{l}(t)$ can be also reconstructed and eliminated. Doing this iterative reconstruction and elimination procedures until only the echo of $s_{N}(t)$ remains in $r_{l}(t)$, the elimination work is done;

3. Reconstruction (the latter one). By multiplying the final eliminated signal with $S_{N}^{*}\left(f_{m}\right)$, we can obtain $\tilde{Y}_{l N}\left(f_{m}\right)$. Applying the same reconstruction work to $\tilde{Y}_{l N}\left(f_{m}\right)$, we have

$$
\tilde{X}_{l N}\left(f_{m}\right)=\sum_{q=1}^{Q_{L N}} d_{l N}^{q}\left(p_{l N}^{q}\right)^{m}+\frac{S_{N}^{*}(m)}{\left|S_{N}(m)\right|^{2}} \delta_{l N}(m)
$$

where $\tilde{X}_{l N}\left(f_{m}\right)=\tilde{Y}_{l N}\left(f_{m}\right) /\left|S_{N}\left(f_{m}\right)\right|^{2}$ and $\delta_{l N}(m)$ contains the noise and eliminating residuals. Since the echo of $\left\{s_{1}(t), \cdots, s_{N-1}(t)\right\}$ has been eliminated from the mixed reception signal $r_{l}(t)$, cross-correlation energy leakage terms in (20) disappear. And the clean signal $\hat{Y}_{1 N}\left(f_{m}\right)$ can be reconstructed by solving all-pole in (20). Essentially, each time for solving all-pole model can be seen as a filtering process, it reserves the signal part while eliminating the disturbance (cross-correlation energy leakage, noise and elimination residuals). Therefore, with this additional reconstruction step, rather than direct MF, this disturbance can be further suppressed. Then, using the inverse fast Fourier transform (IFFT), its corresponding time domain signal $\hat{y}_{1 N}\left(t_{m}\right)$ can be also recovered.

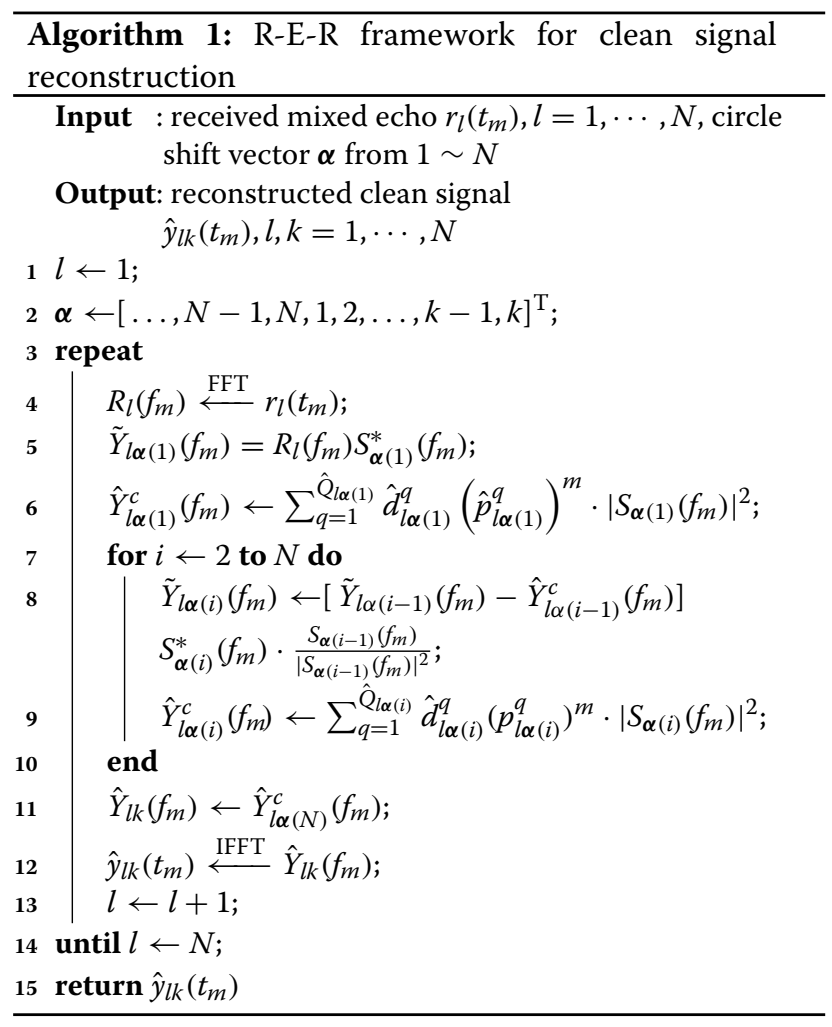

As mentioned above, we only present the clean reconstruction procedures related to $\hat{y}_{1 N}\left(t_{m}\right)$. General reconstruction procedures are outlined as Algorithm 1. Notice that the clean signal cannot be reconstructed in one step and iterative reconstruction and elimination procedures are necessary. Such step-by-step reconstruction and elimination is the major feature of our proposed clean signal reconstruction approach. Particularly, for the dual radar case, the visualized R-E-R framework is shown in Fig. 2. After reconstructing clean echoes $\left\{\hat{y}_{l k}\left(t_{m}\right), l, k=1, \cdots, N\right\}$, CPs will be estimated according to (7), i.e., CC method. For distinction, we refer to

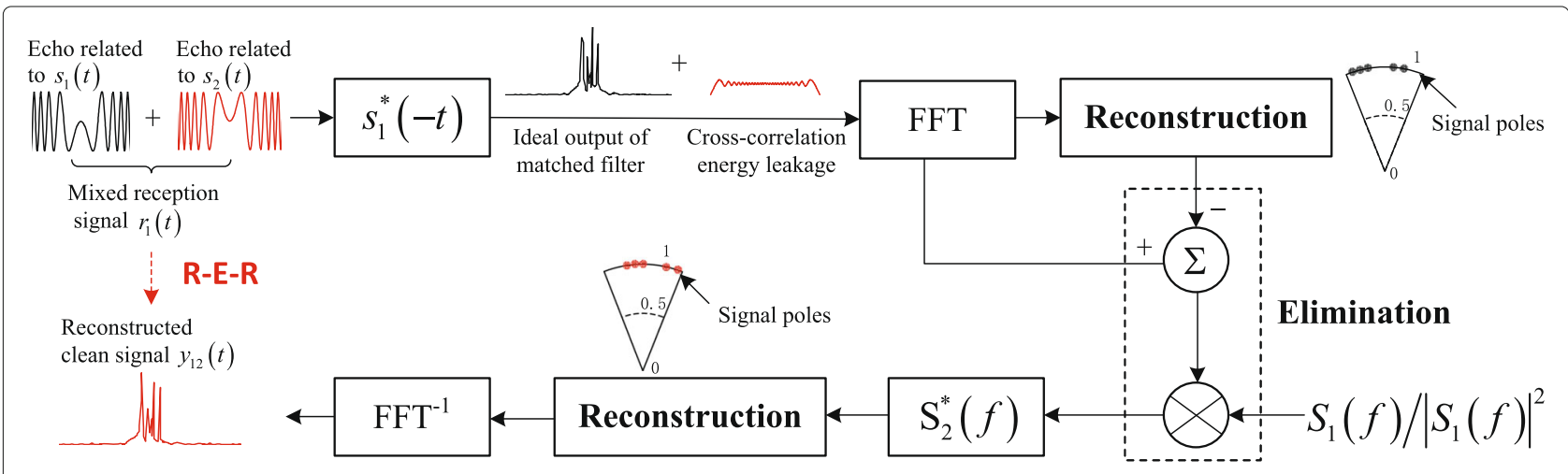

Fig. 2 R-E-R framework for dual radar case 


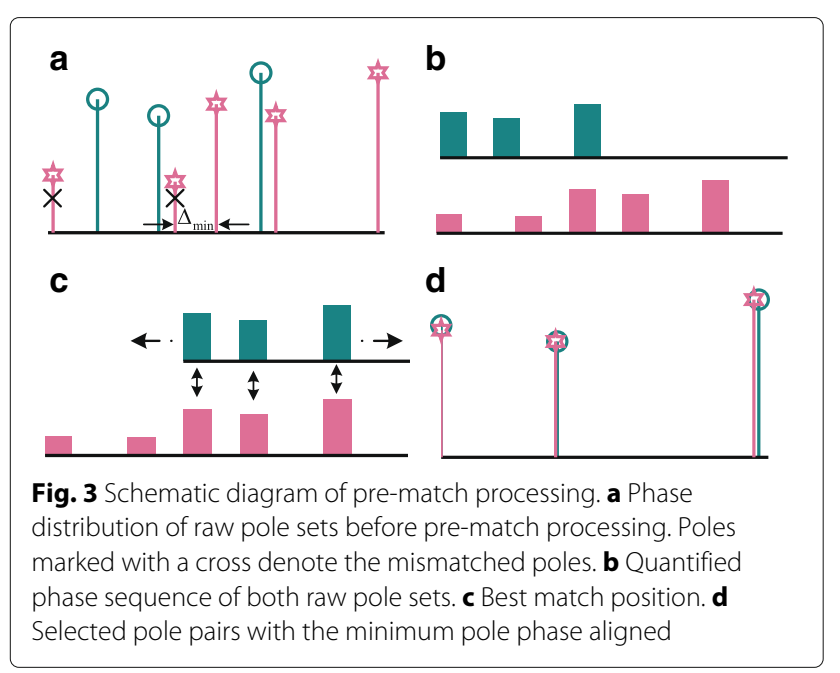

our proposed CP estimation approach as reconstructed cross-correlation (RE-CC) processing.

\subsection{Pre-match processing}

In practice, the number of poles $Q$ is usually unknown. The Akaike Information Criterion (ALC) [18] and minimum description length (MDL) [19] are available to estimate $Q$. But $Q$ estimated in (20) corresponding to different reconstructed clean echoes is not always identical (see Fig. 3a). This is especially true for real measurements characterized by a colored noise and interference. As poles contain the whole information of signal, mismatched poles may cause extra deviation from the true CPs.

Therefore, we propose a method to select the matched pole pairs from raw pole sets as the enhancement of RE$\mathrm{CC}$, called pre-match processing. The selection principle is based on the assumption mentioned earlier; that is, every radar displays same target scattering characteristics. In other words, the relative phase between poles of each reconstructed clean echo is approximately fixed, in spite of disparate absolute pole phase, which can be traced in (16). Assuming pole A and pole B are the raw pole sets stemmed from two different reconstructed clean echoes, the matched pole pairs can be selected by:

1. Quantify. Dividing the pole phase span of poles A and B into several bins, the length of bin $\gamma$ should satisfy $\varepsilon<\gamma<\Delta_{\min }$ to ensure that at most one pole existing in a bin. $\varepsilon$ is the maximum estimated deviation of the pole phase and $\Delta_{\min }$ is the minimum pole phase span of the raw pole sets (see Fig. 3a). And the bin amplitude equals the corresponding pole's normalized amplitude. If no pole falls into a bin, the amplitude is zero (see Fig. 3b).

2. Select. Find the maximum cross-correlation output position of the two quantified sequences as the best match position (see Fig. 3c). Then, we retain the position coincident poles in both sequences as selected pole pairs (see Fig. 3d).

To conclude, combined the R-E-R clean signal reconstruction framework with the pre-matching processing, the overall flow of the RE-CC is shown in Fig. 4.

\section{Simulation results and discussion}

To validate the effectiveness of our proposed approach, we design several simulations in dual radar coherently combining scenario. The dual radar, radar 1 and radar 2 , are located at $(-d / 2,0)$ and $(d / 2,0)$ respectively, where $d=7 \mathrm{~m}$. We choose up- and down-chirp waveforms as the transmitted orthogonal waveform set to separate the

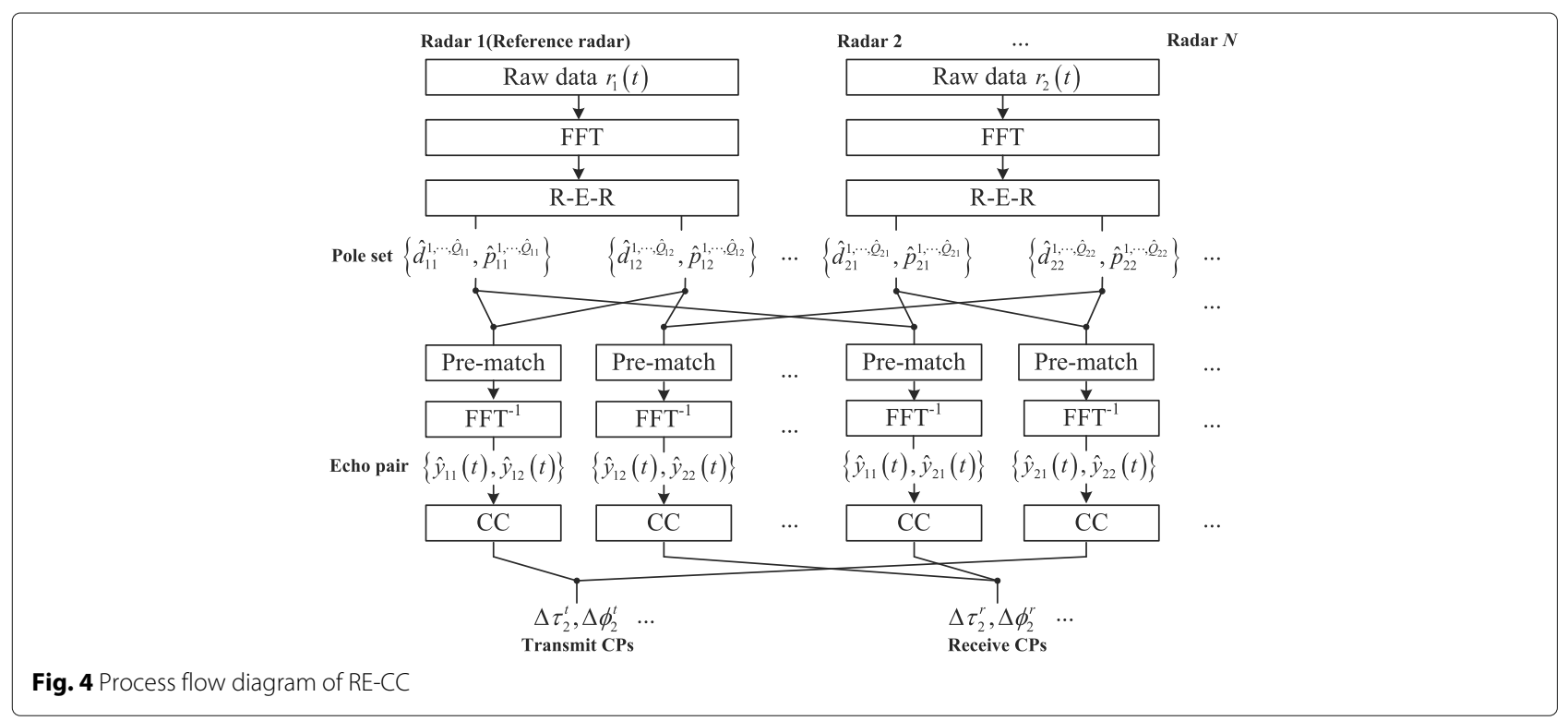


Table 1 Parameters used in simulations

\begin{tabular}{lcc}
\hline Radar & Radar & Radar 2 \\
\hline Signal modulation & Up-chirp & Down-chirp \\
Chirp duration & $1 \mu \mathrm{s}$ \\
Signal bandwidth & $300 \mathrm{MHz}$ \\
Carrier frequency & $10 \mathrm{GHz}$ (X-band) \\
Sampling frequency & $1 \mathrm{GHz}$ \\
Time synchronization error & $0 \mathrm{~s}$ \\
Initial phase & $0 \mathrm{rad}$ \\
Range gate & {$\left[R_{0}-100, R_{0}+100\right] \mathrm{m}$} \\
\hline
\end{tabular}

monostatic and bistatic echoes and estimate CPs. Radar 1 acts as the reference radar and transmits an up-chirp waveform, while radar 2 transmits a down-chirp waveform. Assume that there is a target with five scatterers in the range direction, whose scattering center is located at $\left(R_{0} \cos \theta, R_{0} \sin \theta\right), \theta=60^{\circ}, R_{0}=200 \mathrm{~km}$, and $R_{0} \gg d$. Other parameters used in the simulation are listed in Table 1.

\subsection{CP estimation performance}

According to the discussion in Section 2, in this dual radar coherently combining scenario, the CPs to be estimated are $\left\{\Delta \tau_{2}^{t}, \Delta \tau_{2}^{r}, \Delta \phi_{2}^{t}, \Delta \phi_{2}^{r}\right\}$. In simulations, the effect of noise on CP estimation is further considered. And the noise is added as complex white Gaussian random process, whose intensity is set based on the SNR of a single radar $\left(\mathrm{SNR}_{\mathrm{MF}}^{\mathrm{in}}\right)$. Meanwhile, we assume both radars have the same $\mathrm{SNR}_{\mathrm{MF}}^{\mathrm{in}}$. Besides, for evaluating the CP estimation performance, the root mean square error (RMSE) is introduced, which is defined as (the RMSE of $\Delta \tau_{2}^{t}$ )

$$
\operatorname{RMSE}_{\Delta \tau_{2}^{t}}=\sqrt{\frac{1}{M_{c}} \sum_{i=1}^{M_{c}}\left[\Delta \tau_{2}^{t}(i)-\Delta \hat{\tau}_{2}^{t}(i)\right]^{2}}
$$

where $M_{c}$ is the number of Monte Carlo (MC) runs, $\Delta \tau_{2}^{t}(i)$ and $\Delta \hat{\tau}_{2}^{t}(i)$ are the true and estimated CPs of run $i$. In our simulation, $200 \mathrm{MC}$ runs are executed per each $\mathrm{SNR}_{\mathrm{MF}}^{\mathrm{in}}$ sampled value for each run to capture the average estimating performance.

For comparison, we compare the proposed RE-CC with the CC and the TDM-CC. TDM-CC is the case separating monostatic and bistatic echoes using TDM technique and estimating CPs via CC. To note, when TDM technique is applied, the monostatic and bistatic echoes are staggered in time. And no cross-correlation energy leakages exist in separated echoes, which is equivalent to using ideally orthogonal waveforms. In this case, transmit CP estimation and receive CP estimation are identical. Figure 5 presents the RMSEs for all the three methods.

Noticed that the receive CPs estimated via $\mathrm{CC}$ are asymptotically unbiased and their RMSEs approach the $\mathrm{CRB}$ arbitrarily close in high $\mathrm{SNR}_{\mathrm{MF}}^{\text {in }}$, while the transmit CPs estimated via CC are not, their RMSEs deviate away from the CRB. Also, at the same SNR ${ }_{M F}^{\text {in }}$, the RMSEs of estimated transmit CPs are always greater than those of estimated receive CPs when $\mathrm{CC}$ is used. The reason lies in the existence of cross-correlation energy leakages introduced by non-ideally orthogonal up- and down-chirp waveforms. It is these leakages that cause the difference in transmit and receive CP estimation. Simulation results coincide with the analyses in Section 3.2. Besides, such estimation problems are basically solved by RE-CC, whose performance is comparable to that of TDM-CC, which is the obtainable optimal performance using $\mathrm{CC}$ under the current conditions. Simulation results verify the effectiveness of RE-CC.

To further illustrate the reason behind RE-CC for the enhancement of CP estimation performance, Fig. 6 compares the separated echoes from radar 1 reception before and after clean reconstruction, when $\mathrm{SNR}_{\mathrm{MF}}^{\mathrm{in}}=34.77 \mathrm{~dB}$. As up- and down-chirp waveforms are not ideally
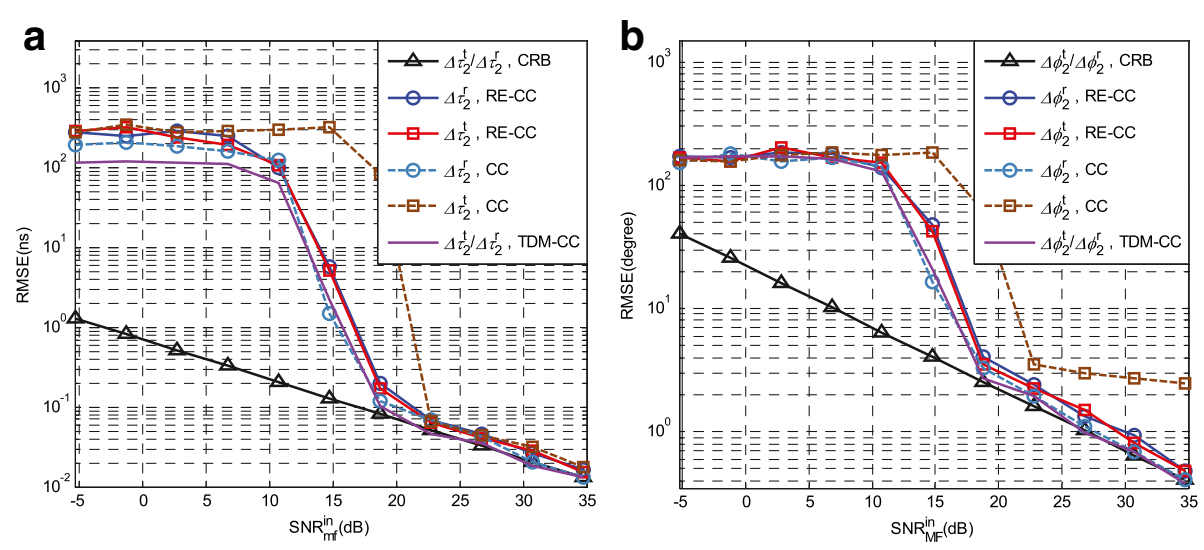

Fig. 5 RMSEs of estimated CPs versus SNR $R_{M F}^{\text {in }}$. a Time CPs. b Phase CPs 


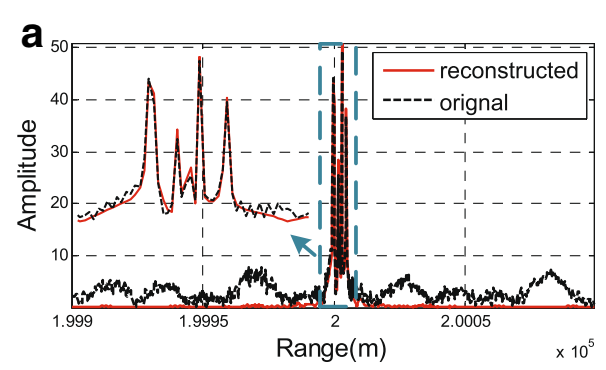

Fig. 6 Separated echoes comparison before and after clean reconstruction.

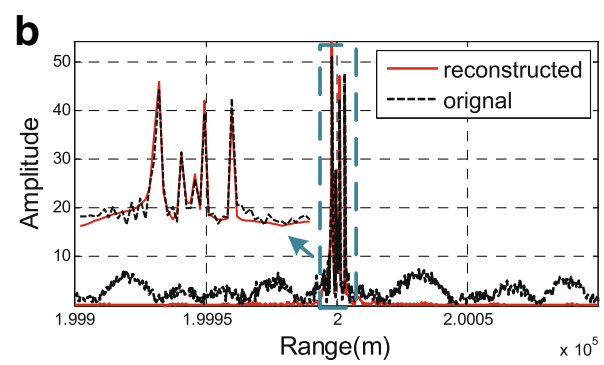

a The echo of up-chirp waveform. $\mathbf{b}$ The echo of down-chirp waveform orthogonal, cross-correlation energy leakages exist before reconstruction, which are presented as fluctuant sidelobes in Fig. 6 (see the black line). Once a clean signal is reconstructed, those sidelobes disappear (see the red line). And RP approximately remains the same, which implies that no information loss occurred. This is the reason why improved CP estimates can be obtained using RE-CC.

\subsection{Dual radar coherent combination performance comparison}

Next, to get a clear understanding of coherently combining benefits, we evaluate the performance of the dual radar system after coherent combination, whose incoherence is calibrated via the CP estimates obtained by RE-CC and $\mathrm{CC}$, respectively. Once $\mathrm{CPs}$ are estimated, to realize constructive interference impinging upon the target, both radars should transmit identical waveform. In our simulation, the up-chirp waveform is transmitted by both radars at this stage. Other parameters remain unchanged. Figure 7 compares the reference and combined signal, when $\mathrm{SNR}_{\mathrm{MF}}^{\text {in }}=18.77 \mathrm{~dB}$. The reference signal is the echo transmitted and received by only radar 1 , while the combined signal is the synthetic echo after dual radar coherent combining.

In Fig. 7a, the dual radar system is combined by RE-CC. Comparing the combined and reference signal, there is a marked drop in noise level for the combined signal. That is, the dual radar system obtains the SNR gain of coherenton-transmit and coherent-on-receive. To evaluate this SNR gain, $200 \mathrm{MC}$ trials are carried out. And the calculated SNR gain is $8.37 \mathrm{~dB}$, approaching the ideal level $9 \mathrm{~dB}$ $\left(10 \log 2^{3}\right)$. By contrast, in Fig. $7 b$, the dual radar system is combined via CC. Compared to the reference signal, a slight noise level drop still exists in the combined signal. But RPs of the two signals no longer overlap. In this case, the SNR gain is also calculated, which is $4.52 \mathrm{~dB}$. Apparently, under this situation, using RE-CC can obtain higher SNR gain than CC.

Furthermore, from these simulation results, it can be inferred that using the approach transmitting orthogonal waveforms and estimating CPs to coherently combine multiple radars is conditional. Once the SNR of single radar is too low, the mutual incoherence among radars cannot be calibrated via the inaccurate estimated CPs. In this case, coherently combining radars is in vain.

\section{Conclusions}

In this paper, we proposed a novel approach (RE-CC) for coherently combining multiple radars, based on a clean signal reconstruction framework (R-E-R). This method mainly solves the impaired estimation performance of CPs resulting from transmitting non-orthogonal waveforms. Theoretical analyses and simulation results indicate that the influence of transmitting non-orthogonal waveforms
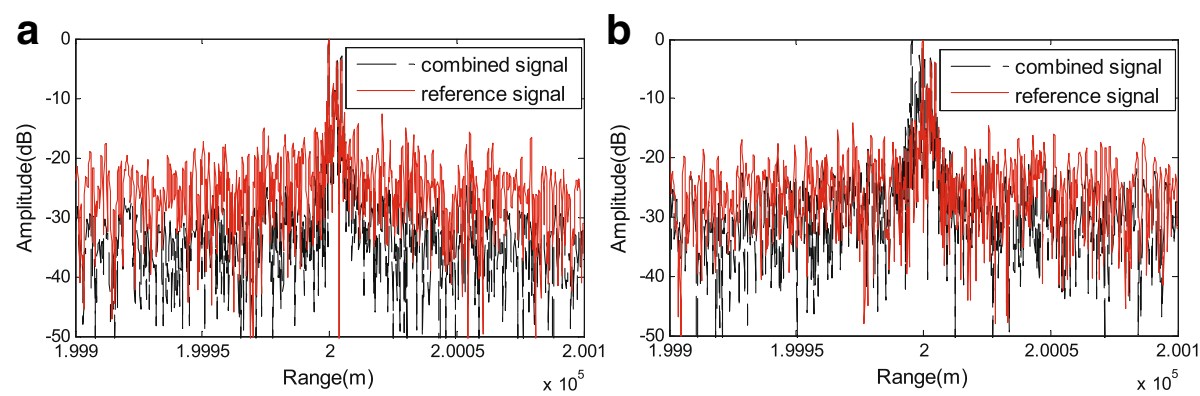

Fig. 7 Comparison between the reference and combined signal. a Dual radar coherent combination realized by RE-CC. b Dual radar coherent combination realized by CC 
on $\mathrm{CP}$ acquisition can be approximately ignored after employing RE-CC. In addition, further comparisons are carried out on dual radar coherent combination performance. Results show that, under the same conditions, using RE-CC can obtain higher coherent combination SNR gain than using CC.

Furthermore, it is worth to note that the application of the R-E-R framework is not limited to reconstruct the clean signal from the signal mixed with up- and downchirp waveforms and, for a signal mixed with general orthogonal waveforms, e.g., the orthogonal phase-coding waveforms, it is also effective. Meanwhile, in contrary to the perfect orthogonal waveform design, R-E-R framework offers an alternative attempt for cross-correlation energy leakage suppression, which is expected to be extended into much wider radar applications. Besides, when the target is moving, CPs estimated via the previous pulse will no longer adapt the pulse next. Therefore, how to predict the CPs to adapt the successive pulse will be an interesting subject for future research.

\section{Appendix A: Expression of $g_{k}(t, \tau)$ in (10)}

In this appendix, we give the detailed expression of $g_{k}(t, \tau)$. According to (6) and $\Delta \tau_{1}^{t}=\Delta \phi_{1}^{t}=0$, we have

$$
\begin{aligned}
y_{l 1}(t-\tau)= & \xi A_{1}^{\prime}\left(t-\tau_{11}-\Delta \tau_{l}^{r}-\tau\right) e^{j \Delta \phi_{l}^{r}} \\
& +\xi \sum_{k^{\prime}=2}^{N} C_{k^{\prime} 1}^{\prime}\left(t-\tau_{11}-\Delta \tau_{k^{\prime}}^{t}-\Delta \tau_{l}^{r}-\tau\right) e^{j \Delta \phi_{k^{\prime}}^{t}+j \Delta \phi_{l}^{r} .}
\end{aligned}
$$

Since $\int_{T_{\mathrm{G}}} y_{l k}(t) y_{l 1}^{*}(t-\tau) d t=\int_{T_{\mathrm{G}}} y_{l k}\left(t+\Delta \tau_{l}^{r}\right) y_{l 1}^{*}$ $\left(t+\Delta \tau_{l}^{r}-\tau\right) d t$ holds, we can express $x_{k, l}^{t}(\tau)$ in the following simplified form $\left(|\xi|^{2}\right.$ is omitted)

$$
\begin{aligned}
x_{k, l}^{t}(\tau) & =\int_{T_{\mathrm{G}}} y_{l k}(t) y_{l 1}^{*}(t-\tau) d t \\
& =\int_{T_{\mathrm{G}}} e^{j \Delta \phi_{k}^{t}} A_{k}^{\prime}\left(t-\tau_{11}-\Delta \tau_{k}^{t}\right)\left[A_{1}^{\prime}\left(t-\tau_{11}-\tau\right)\right]^{*}+g_{k}(t, \tau) d t
\end{aligned}
$$

where

$$
\begin{aligned}
g_{k}(t, \tau)= & A_{k}^{\prime}\left(t-\tau_{11}-\Delta \tau_{k}^{t}\right) e^{j \Delta \phi_{k}^{t}}\left[\sum_{k^{\prime}=2}^{N} C_{k^{\prime} 1}^{\prime}\left(t-\tau_{11}-\Delta \tau_{k^{\prime}}^{t}-\tau\right) e^{j \Delta \phi_{k^{\prime}}^{t}}\right]^{*} \\
& +\sum_{\substack{k^{\prime}=1 \\
k^{\prime} \neq k}}^{N} C_{k^{\prime} k}^{\prime}\left(t-\tau_{11}-\Delta \tau_{k^{\prime}}^{t}\right) e^{j \Delta \phi_{k^{\prime}}^{t}}\left[A_{1}^{\prime}\left(t-\tau_{11}-\tau\right)\right]^{*} \\
& +\sum_{\substack{k^{\prime}=1 \\
k^{\prime} \neq k}}^{N} C_{k^{\prime} k}^{\prime}\left(t-\tau_{11}-\Delta \tau_{k^{\prime}}^{t}\right) e^{j \Delta \phi_{k^{\prime}}^{t}}\left[\sum_{k^{\prime}=2}^{N} C_{k^{\prime} 1}^{\prime}\left(t-\tau_{11}-\Delta \tau_{k^{\prime}}^{t}-\tau\right) e^{j \Delta \phi_{k^{\prime}}^{t}}\right]^{*}
\end{aligned}
$$

\section{Appendix B: Procedures for solving all-pole model} The all-pole model in (16) can be solved with the following four-step process:
1 Use the sampling data $X_{l 1}(m)$ to construct the Hankel matrix

$$
\mathbf{H}_{l 1}=\left[\begin{array}{cccc}
X_{l 1}(0) & X_{l 1}(1) & \cdots & X_{l 1}(M-L) \\
X_{l 1}(1) & X_{l 1}(2) & \cdots & X_{l 1}(M-L+1) \\
\vdots & \vdots & \ddots & \vdots \\
X_{l 1}(L-1) & X_{l 1}(L) & \cdots & X_{l 1}(M-1)
\end{array}\right]
$$

where $L$ is the length of the correlation window length. Generally, $L=\lceil M / 3\rceil$;

2 Applying the singular-value decomposition (SVD) to express $\mathbf{H}_{l 1}=\mathbf{U}_{l 1} \mathbf{S}_{l 1} \mathbf{V}_{l 1}^{\mathrm{H}}$, and the model order $Q_{l 1}$ can be determined by the decomposed singular-value matrice $\mathbf{S}_{l 1}$;

3 According to the estimated $\hat{Q}_{l 1}, \mathbf{U}_{l 1}$, and $\mathbf{V}_{l 1}$, signal or noise subspace can be built. On the basis of these subspaces, the estimation of signal parameters via rotational invariance techniques (ESPRIT) [20] and the root multiple signal classification (root-MUSIC) [21] are available to estimate poles $p_{l 1}^{q}, q=1, \cdots, \hat{Q}_{l 1}$;

4. Once the poles are known, the amplitude terms $d_{l 1}^{q}, q=1, \cdots, \hat{Q}_{l 1}$ are estimated by fitting the all-pole model to the data $X_{l 1}(m)$ using a linear least-squares(LLS) or a non-linear least-squares (NLLS) algorithm.

\section{Abbreviations}

ALC: Akaike Information Criterion; CC: Cross-correlation; CPs: Coherent parameters; CRB: Cramer-Rao bound; ESPRIT: Estimation of signal

parameters via rotational invariance techniques; GDT: Geometrical diffraction theory; IFFT: Inverse fast Fourier transform; LLS: Linear least-squares; LOS: Line of sight; MDL: Minimum description length; MF: Matched filtering; NLLS: Non-linear least-squares; RE-CC: Reconstructed cross-correlation; R-E-R: Reconstruction-elimination-reconstruction; RF: Radio frequency; RP: Range profile; RMSE: Root mean square error; root-MUSIC: Root multiple signal classification; SNR: Signal-to-noise ratio; SVD: Singular-value decomposition; TDM: Time-division multiplexing

\section{Acknowledgements}

The authors would like to thank the anonymous reviewers for their valuable comments and suggestions.

\section{Funding}

This work was supported in part by the National Natural Science Foundation of China under Grant 61471372.

Availability of data and materials

Please contact author for data requests.

Authors' contributions

All the authors have participated in writing the manuscript and have revised the final version. All authors read and approved the final manuscript.

\section{Competing interests}

The authors declare that they have no competing interests.

\section{Author details}

${ }^{1}$ State Key laboratory of CEMEE, National University of Defence Technology, Yanwachi 137, Changsha, People's Republic of China. ${ }^{2}$ Electronic Engineering Institute, Huangshan Road 460, 230037 Hefei, People's Republic of China. 


\section{Publisher's Note}

Springer Nature remains neutral with regard to jurisdictional claims in published maps and institutional affiliations.

Received: 18 January 2018 Accepted: 18 June 2018

Published online: 18 July 2018

\section{References}

1. GD Thome, RP Enzmann, F Steudel, System and method for coherently combining a plurality of radars. (Google Patents, 2007). EP Patent App. EP20,060,738,577. https://encrypted.google.com/patents/EP1869492A1? $\mathrm{Cl}=$ und

2. AS Fletcher, FC Robey, in Proceedings of the 12th Annual Workshop on Adaptive Sensor Array Processing. Performance bounds for adaptive coherence of sparse array radar (Massachusetts inst of tech Lexington Lincoln lab, Lexington, 2003)

3. KM Cuomo, Lincoln Laboratory, Wideband Aperture Coherence Processing for Next Generation Radar (NexGen). Project report NG. (Massachusetts Institute of Technology, Lincoln Laboratory, 2004). https://books.google. gr/books?id=GvdFtwAACAAJ

4. E Brookner, DV Manoogian, F Steudel, Multiple radar combining for increased range, radar sensitivity and angle accuracy. (Google Patents, 2005). US Patent 6,977,610

5. S Coutts, K Cuomo, J McHarg, F Robey, D Weikle, in Sensor Array and Multichannel Processing, 2006. Fourth IEEE Workshop On. Distributed coherent aperture measurements for next generation bmd radar (IEEE, Waltham, 2006), pp. 390-393

6. Y Vagman, System and method for coherent processing of signals of a plurality of phased arrays. (Google Patents, 2016). US Patent 9,496,611

7. P Sun, J Tang, Q He, B Tang, X Tang, Cramer-Rao bound of parameters estimation and coherence performance for next generation radar. IET Radar, Sonar Navig. 7(5), 553-567 (2013)

8. P Sun, J Tang, X Tang, Cramer-Rao bound and signal-to-noise ratio gain in distributed coherent aperture radar. J. Syst. Eng. Electron. 25(2), 217-225 (2014)

9. X Tang, J Tang, Q He, S Wan, B Tang, P Sun, N Zhang, Cramér-Rao bounds and coherence performance analysis for next generation radar with pulse trains. Sensors. 13(4), 5347-5367 (2013)

10. T Zeng, P Yin, Q Liu, Wideband distributed coherent aperture radar based on stepped frequency signal: theory and experimental results. IET Radar, Sonar Navig. 10(4), 672-688 (2016)

11. P Yin, X Yang, Q Liu, T Long, in Radar Conference, 2014 IEEE. Wideband distributed coherent aperture radar (IEEE, Cincinnati, 2014), pp. 1114-1117

12. $P$ Yin, $X$ Yang, $T$ Zeng, $X$ Hu, in Phased Array Systems \& Technology, 2013 IEEE International Symposium On. Robust time synchronization method based on step frequency signal for wideband distributed coherent aperture radar (IEEE, Waltham, 2013), pp. 383-388

13. H-W Gao, Z Cao, Y-b Lu, P-X Wang, in Radar Conference 2013, IET International. Development of distributed aperture coherence-synthetic radar technology (IET, Xi'an, 2013), pp. 1-6

14. H Gao, B Zhou, D Zhou, Z Jin, in 2016 CIE International Conference on Radar (RADAR). Performance analysis and experimental study on distributed aperture coherence-synthetic radar (Institute of Electrical and Electronics Engineers Inc., Guangzhou, 2016), pp. 1-5

15. KM Cuomo, JE Pion, JT Mayhan, Ultrawide-band coherent processing. IEEE Trans. Antennas Propag. 47(6), 1094-1107 (1999)

16. H Borrion, H Griffiths, P Tait, D Money, C Baker, in Radar Conference, 2005 IEEE International. Scattering centre extraction for extended targets (IEEE, Arlington, 2005), pp. 173-178

17. B Tian, Z Chen, S Xu, Sparse subband fusion imaging based on parameter estimation of geometrical theory of diffraction model. IET Radar, Sonar Navig. 8(4), 318-326 (2013)

18. E Fishler, M Grosmann, H Messer, Detection of signals by information theoretic criteria: General asymptotic performance analysis. IEEE Trans. Signal Process. 50(5), 1027-1036 (2002)
19. M Wax, I Ziskind, Detection of the number of coherent signals by the $\mathrm{mdl}$ principle. IEEE Transactions on Acoust. Speech Signal Process. 37(8), 1190-1196 (1989)

20. R Roy, T Kailath, Esprit-estimation of signal parameters via rotational invariance techniques. IEEE Trans. Acoust. Speech Signal Process. 37(7), 984-995 (1989)

21. M Jalali, MN Moghaddasi, A Habibzadeh, in Microwave Symposium (MMS), 2009 Mediterrannean. Comparing accuracy for ml, music, root-music and spatially smoothed algorithms for 2 users (IEEE, Tangiers, 2009), pp. 1-5

\section{Submit your manuscript to a SpringerOpen ${ }^{\circ}$ journal and benefit from:}

- Convenient online submission

- Rigorous peer review

- Open access: articles freely available online

- High visibility within the field

- Retaining the copyright to your article

Submit your next manuscript at $\$$ springeropen.com 